\title{
可见光催化脱羧偶联反应研究进展
}

\author{
关保川许孝良*王 红* 李小年 \\ (浙江工业大学 化学工程学院 杭州 310014)
}

\begin{abstract}
摘要 可见光介入的偶联反应近年来得到了广泛的关注，已经发展成 $\mathrm{C}-\mathrm{H}$ 活化、构建新型 $\mathrm{C}-\mathrm{C} 、 \mathrm{C}-\mathrm{X}$ 键的重要方 法之一. 可见光催化体系的发展也为脱羧偶联反应提供了新的手段, 许多关于可见光催化的脱羧反应研究见诸报端. 对近年来可见光催化的脱羧反应进行了简要概述.
\end{abstract}

关键词 可见光催化; 脱羧; 偶联反应

\section{Progress on the Decarboxylation Coupling Reaction Mediated by Visible Light}

\author{
Guan, Baochuan Xu, Xiaoliang* Wang, Hong* Li, Xiaonian \\ (College of Chemical Engineering, Zhejiang University of Technology, Hangzhou 310014)
}

\begin{abstract}
Visible light mediated coupling reaction has obtained the widespread attention in recent years, and has been a most efficient method for building new $\mathrm{C}-\mathrm{C}$ and $\mathrm{C}-\mathrm{X}$ bonds. The development of visible light catalytic system also has provided a new means for decarboxylation reaction. Lots of studies on visible light catalytic decarboxylation reaction were published recently. In this paper, the visible light mediated decarboxylation reactions in recent years are briefly reviewed.

Keywords visible light catalysis; decarboxylation; coupling reaction
\end{abstract}

羧酸化合物具有廉价、易得、稳定等特点, 广泛存 在于自然界中 ${ }^{[1]}$. 利用羧酸作为起始原料合成一系列人 类生活所需的物质, 如药物、功能材料等是一个热门的 研究领域. 脱羧偶联是有机合成中一种非常重要的化学 转化，常用来构建新的 $\mathrm{C}-\mathrm{C}$ 键和 $\mathrm{C}-\mathrm{X}$ 键. 近年来, 以 过渡金属催化的脱羧偶联反应取得了较大的进展 ${ }^{[2 \sim 5]}$. 可见光催化方法学是近几年来发展起来的一种强大的 合成手段, 以其低能耗、无污染和原子经济性等特点而 广受关注, 给有机合成化学带来新的春天 ${ }^{[6,7]}$. MacMil$\operatorname{lan}^{[8 \sim 13]} 、 Y^{20 o n}{ }^{[14 \sim 18]} 、 S t e p h e n s o n{ }^{[19 \sim 22]} 、 X i a 0^{[23 \sim 25]}$ 等课 题组近年来在该领域做了大量的开创性工作, 使得可见 光催化得到了迅速的发展. 可见光催化的发展为脱羧偶 联反应提供了新的方法, 尤其最近将过渡金属引入到光 催化体系中组成双催化体系, 发展了羧酸类化合物参与 的自由基反应模式, 大大扩展了羧酸功能化反应的类型 [26,27]. 自 2008 年以来越来越多的化学工作者开展了相 关的研究工作, 并取得了较好的效果, 本文对近年来可
见光介入的脱羧偶联反应的研究做了简单概述.

\section{1 芳基化反应}

$\alpha$-氨基酸广泛存在于自然界中, 具有生物活性的氨 基酸是合成医药、生物质等物质的重要原料. 2014 年 MacMillan 课题组 ${ }^{[28]}$ 报道了可见光催化的 Boc- $L$-脯氨酸 与对苯二腈的脱羧偶联反应(Scheme 1). 该反应在较为 温和的条件下实现了 $\alpha$-氨基酸的脱羧芳基化反应，为合 成一系列具有生物活性的伯胺、茮基胺等医药中间体提 供了新的方法.

在对该反应的探讨中，作者通过对光催剂配体的修 饰来调控催化剂的氧化还原能力从而实现脱羧氧化和 芳腈的还原两个过程都能高效的进行(图 1). 在可见光 的激发下，基态的三价铱催化剂通过金属向配体的电子 转移(MLCT)过程变成有较高还原能力的激发态的三价 铱, 与对苯二腈发生一个单电子转移过程将 2 还原到自 由基阴离子 5, 同时铱催化剂被氧化到四价，具有强氧

\footnotetext{
*E-mail: xuxiaoliang@zjut.edu.cn; chem hong@163.com

Received January 11, 2016; revised February 17, 2016; published online March 11, 2016.

Project supported by the Natural Science Foundation of Zhejiang Province (No. LY15B020004).

浙江省自然科学基金(No. LY15B020004)资助项目.
} 
化能力的四价铱进一步氧化羧酸脱羧生成自由基 4 , 与 此同时催化剂被还原到三价完成一个催化循环, 随后 4 和 5 通过自由基偶联生成中间体 $\mathbf{6}$, 进一步失去 $\mathrm{CN}^{-}$生 成目标产物 3.

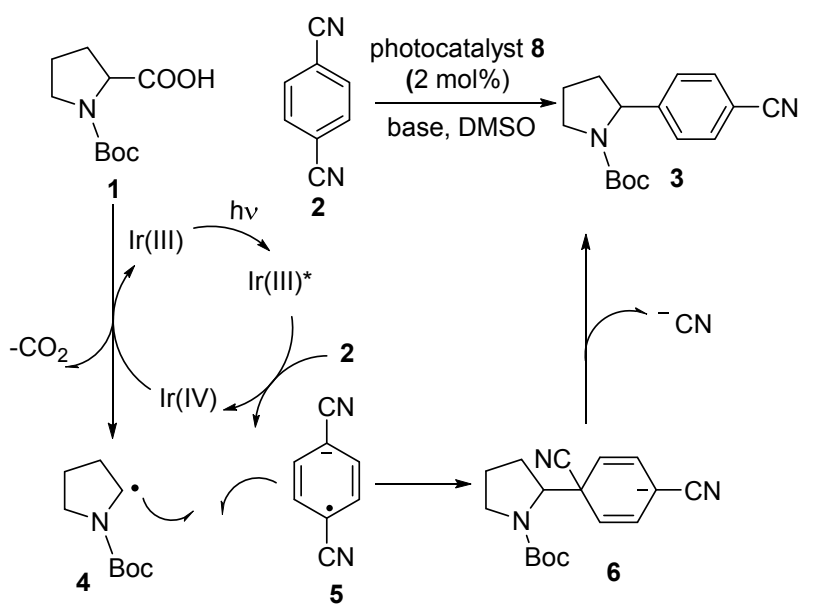

图式 1 可见光催化的羧酸与芳腈的脱羧偶联反应

Scheme 1 Reaction of carboxylic acid and aryl cyanide catalyzed by visible light

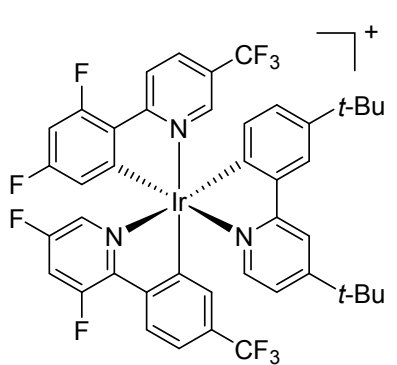

$\operatorname{Ir}\left[\mathrm{dF}\left(\mathrm{CF}_{3}\right) \mathrm{ppy}\right]_{2}(\mathrm{dtbbpy})(\mathbf{7})$

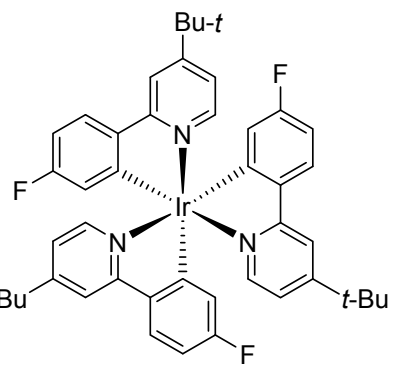

$\operatorname{Ir}[p-\mathrm{F}(t-\mathrm{Bu})-\mathrm{ppy}]_{3}(\mathbf{8})$
图 1 两种光催化剂的结构

Figure 1 Structures of two kinds of photocatalyst

过渡金属催化交叉偶联反应是有机合成中构建新 型 $\mathrm{C}-\mathrm{C}, \mathrm{C}-\mathrm{N}, \mathrm{C}-\mathrm{O}$ 等重要的方法之一. 近年来将过 渡金属引入可见光催化方法中构成双催化体系的报道 屡见报端 ${ }^{[29-35]}$. 传统的过渡金属催化脱羧以其高的效 率得到了广大化学工作者的关注 ${ }^{[36 ~ 39]}$. 将过渡金属引 入光催化脱羧偶联反应中给那些在单催化体系中难以 实现的反应提供了新的思路. 2014 年, Zuo 等 ${ }^{[40]}$ 报道了 过渡金属镍与光催化剂组成的双催化体系催化氨基酸 脱羧与卤代芳烃的偶联(Eq. 1). 相对于上述的研究工作, 该反应的底物范围进一步扩大, 不仅当卤代芳烃的芳环 上连有缺电子基团时能反应, 连有甲基、甲氧基等富电 子基团时仍然得到了目标产物, 而且收率较高. 过渡金 属的引入解决了像卤代芳烃、烷烃、烯烃等物质难以在 光催化体系中活化的问题, 广泛的应用在构建新型 $\mathrm{C}_{\mathrm{sp}^{3}}-\mathrm{C}_{\mathrm{sp}^{2}}, \mathrm{C}_{\mathrm{sp}^{3}}-\mathrm{C}_{\mathrm{sp}^{3}}$ 上.

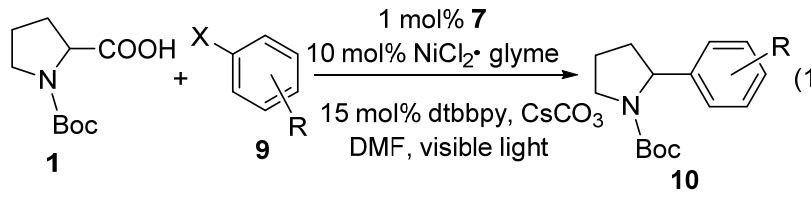

该反应过程如 Scheme 2 所示, 在可见光激发作用 下，基态的三价铱通过 MLCT 过程产生激发态的三价 铱，具有强氧化性的三价铱氧化羧酸脱去 $\mathrm{CO}_{2}$ 生成烷基 自由基 4, 同时催化剂被还原到二价; 另一边零价的 $\mathrm{Ni}$ 与卤代芳烃氧化加成生成 $\mathrm{Ni}^{\mathrm{II}}$ 中间体 $\mathbf{1 1}$, 进一步捕捉自 由基 4 生成 $\mathrm{Ni}^{\mathrm{III}}$ 物种, 再经还原消除生成目标产物 $\mathbf{1 0}$ 和 $\mathrm{Ni}^{\mathrm{I}} 13$, 最后 13 被二价铱还原到零价, 光催化剂被氧 化到三价，两个催化循环同时完成.

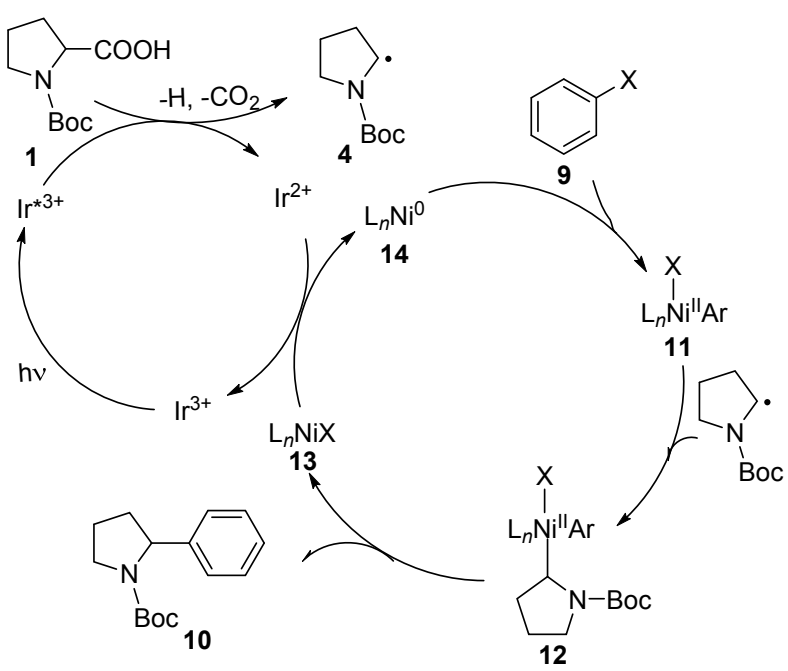

图式 2 双催化体系反应机理

Scheme 2 Reaction mechanism of the dual catalytic system

随后 Oderinde 等 ${ }^{[4]}$ 考查了这种双催化体系的影响 因素并重现了上述反应,再一次证明了该方法的可行性, 通过实验发现，分子氧能促进 $\mathrm{Ni}(\mathrm{II})$ 还原到 $\mathrm{Ni}(0)$ 的过程， 提出了氧气是该实验关键的影响因素.

MacMillan 课题组 ${ }^{[42]}$ 随后报道了酮酸的脱羧芳基化 反应，由酮酸脱羧生成酰基自由基这一中间体合成芳基 酮(Eq. 2). 酮酸的底物范围包括芳基酮酸和脂肪酮酸, 而且磑代芳烃和溴代芳烃都能很好的参与反应. 值得一 提的是，在对该反应的延伸实验中，作者利用澳代烯烃 和溴代烷烃作为亲电试剂在该体系中成功合成了 $\alpha, \beta$-不 饱和酮和二烷基酮. 为了证明该方法的应用价值，作者 将这一方法应用在胆固醇调剂药物非诺贝特的合成并 得到了较好的效果(Eq. 3).

$\mathrm{Fu}$ 等 ${ }^{[43]}$ 将该反应的催化体系由 $\mathrm{Ni}$ 扩大到 $\mathrm{Pd}$ ，同样 取得了较好的收率，而且底物范围扩到酰胺的羧酸盐， 也进一步证明了该方法的普适性(Eq. 4). Ni，Pd 等过渡 


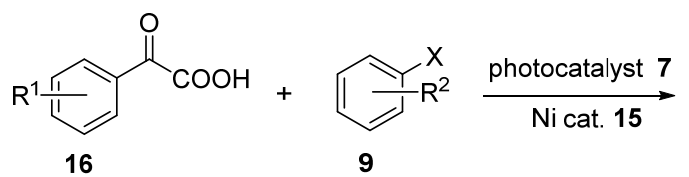<smiles>[R][R]1ccc(C(=O)c2cc[R1]cc2)cc1</smiles><smiles>CC(C)OC(=O)C(C)(C)Oc1ccc(C(=O)C(=O)O)cc1</smiles>
19<smiles>CC(C)OC(=O)C(C)(C)Oc1ccc(C(=O)c2ccc(Cl)cc2)cc1</smiles>

金属催化剂的引入不仅扩大了光催化体系的应用, 而且 克服了传统脱羧芳基化反应需要高温条件的缺点.

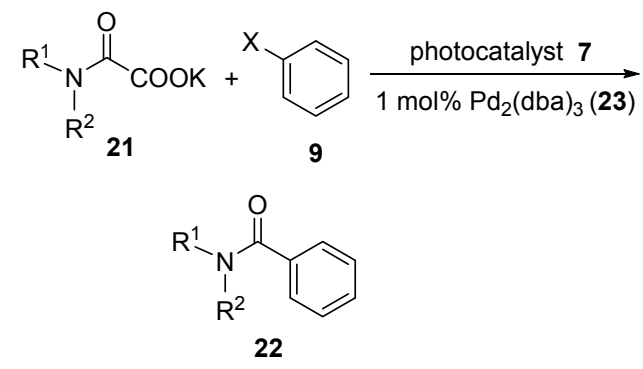

\section{2 烯基化反应}

上述芳基化反应中提到过渡金属与光催化剂组成 的双催化体系在脱羧芳基化反应中得到了很好的应用， 也为其他类型的反应提供了新的参考. 2014 年 MacMillan 课题组 ${ }^{[44]}$ 报道了将该催化体系用来催化羧酸 脱羧与卤代烯烃的偶联反应(Eq. 5). 通过两种催化剂的 协同作用, 实现了底物的烯基化, 构建了新型 $\mathrm{C}_{\mathrm{sp}^{3}}$ $\mathrm{C}_{\mathrm{sp}^{2}}$, 为制备官能化的烯烃提供了新的方法. 含氧羧酸、 含氮羧酸及碳氢取代的羧酸都适用于该体系, 而且不同 碘代、溴代烯烃都能参与反应.

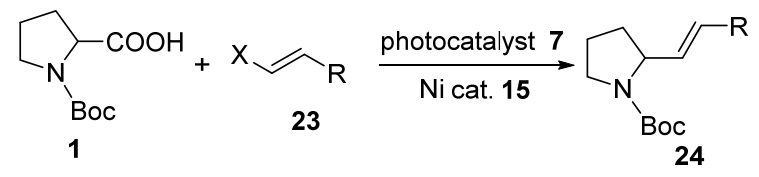

吲哚及其衍生物是一类重要的化工原材料, 广泛存 在于天然产物中如茉莉花、煤焦油等, 同时也是很多药 物的核心结构, 例如止痛药普拉朵林等. 因此关于吲哚
及其衍生物的研究具有重要的意义. 在可见光催化体系 中有关这类化合物的合成研究也有很多报道 ${ }^{[45 \sim 53]}, \mathrm{Gu}$ 课题组 ${ }^{[54]}$ 在 2015 年报道了在光催化体系中利用酮酸脱 羧与吲哚偶联反应合成 3-酰基吲哚衍生物的研究, 如图 示(Eq. 6). 该反应条件温和，底物范围广泛，为合成系 列功能化的 3-酰基吲哚提供了新的方法. 同时这也证明 了该双催化体系在 $\mathrm{C}-\mathrm{C}$ 键和 $\mathrm{C}-\mathrm{H}$ 键活化中的适用性.

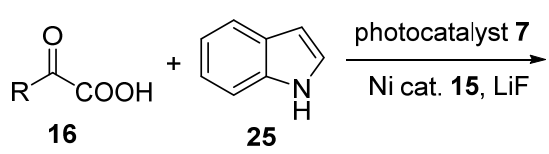<smiles>[R]C(=O)c1c[nH]c2ccccc12</smiles>

除了引入过渡金属来实现烯基化以外, MacMillan 课题组 ${ }^{[55}$ 通过利用带有砜基的烯烃与 $\alpha$-氨基羧酸在可 见光催化体系中通过 $\beta$-消除来实现烯基化反应合成一 系列的烯基胺(Eq. 7).

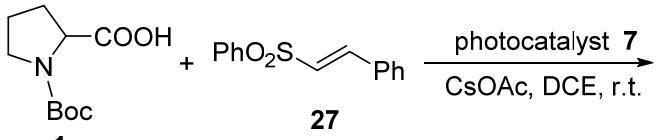

1<smiles>O=C(O)N1CCCC1/C=C/c1ccccc1</smiles>

在可见光作用下，三价铱催化剂被激发到具有较高 氧化能力的激发态， 1 首先被氧化脱除 $\mathrm{CO}_{2}$ 生成自由基 中间体 4, 催化剂则被还原到二价, 4 与 27 作用生成 $\beta$ 砜基自由基 29, 随后通过消除砜基自由基生成目标产 物 28, 同时砜基自由基 30 被还原到砜基阴离子 $\mathbf{3 1}$,二价 铱被氧化到三价完成一个催化循环(Scheme 3).

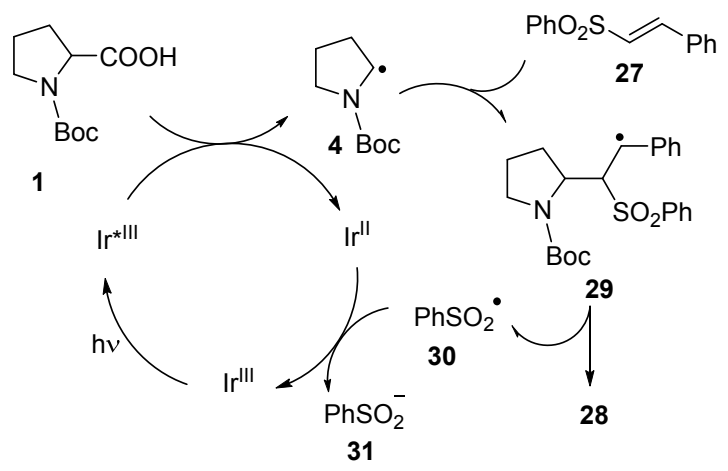

图式 $3 \alpha$-砜基烯烃与氨基酸的反应机理

Scheme 3 Reaction mechanism of $\alpha$-sulfonyl vinyl and carboxylic acid

高价碘是一种常用的氧化剂，它具有类似于过渡金 属的反应活性和和对环境友好等特点, 近年来, 在有机 
合成化学中备受瞩目. Chen 等 ${ }^{[56]}$ 在 2015 年首次在可见 光催化体系中利用高价碘试剂促进烯基羧酸的脱羧偶 联反应合成官能化的烯烃, 如图示(Eq. 8). 与传统的过 渡金属催化的烯基酸脱羧反应相比, 该方法条件更为温 和，室温条件下即可达到较高的化学选择性和收率. 该 反应中高价碘试剂的成功应用也为后续的研究奠定了 基础.

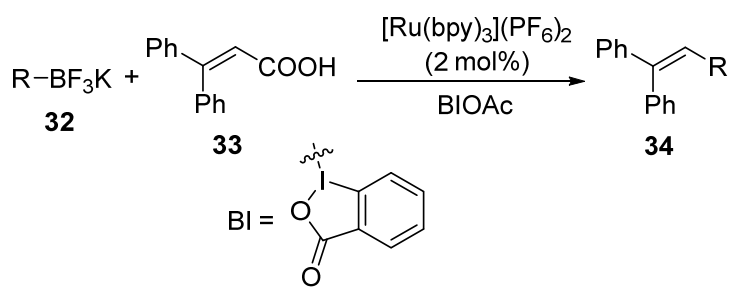

\section{3 炔基化反应}

炔基化是有机合成中重要的化学转变之一, 早期在 可见光催化体系中构建新型 $\mathrm{C}_{\mathrm{sp}^{3}}-\mathrm{C}_{\mathrm{sp}}$ 键和 $\mathrm{C}_{\mathrm{sp}^{2}}-\mathrm{C}_{\mathrm{sp}}$ 键 的研究中已有相关报道 ${ }^{[57 ~ 59]}$, 而这些工作大部分都是 引入 $\mathrm{Cu} 、 \mathrm{Au}$ 等过渡金属来实现炔基化反应. 如前所述, MacMillan 课题组利用烯基砜实现羧酸的脱羧烯基化反 应，而 Chen 等 ${ }^{[60]}$ 在 2015 年报道了炔基砜在可见光催化 体系中与羧酸的偶联实现炔基化反应(Eq. 9).<smiles>[R]CC(=O)ON1C(=O)c2ccccc2C1=O</smiles>

该反应不仅条件简单温和而且反应时间只有 $0.5 \mathrm{~h}$, 在有机溶剂和中性水溶液中都能很好的反应. 作者也尝 试了生物大分子在这种体系下的炔基化反应得到了较 好的效果. 但是该反应仅限制在邻苯二甲酰亚胺取代的 羧酸酯参与炔基化的过程, 底物的范围较小, 而且高价 碘取代的炔试剂在该条件下受到一定的限制。该小 组 ${ }^{[61]}$ 后续的研究工作不仅将邻苯二甲酰亚胺取代的酯 底物范围扩大到酮酸，而且高价碘取代的炔也表现出了 较高的反应活性(Eq. 10).

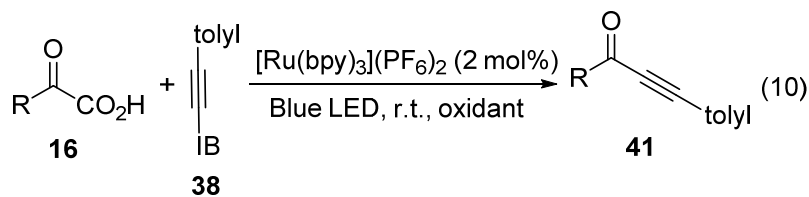

该反应首次以高价碘 BI-OAc 作为氧化剂, 通过与 光催化剂的协同作用而合成羰基炔(Scheme 4). 酮酸 16 与氧化剂 BI-OAc 作用生成中间体 39, 紧接着再被氧化 性强的三价钓氧化生成酰基自由基和 BI-OAc, 完成高
价碘的催化循环; 酰基自由基进攻炔 38 生成烯基自由 基 40 再经离去 $\mathrm{BI}$ 自由基生成羰基炔，另一边 $\mathrm{BI}$ 自由基 被还原到阴离子，完成催化循环.

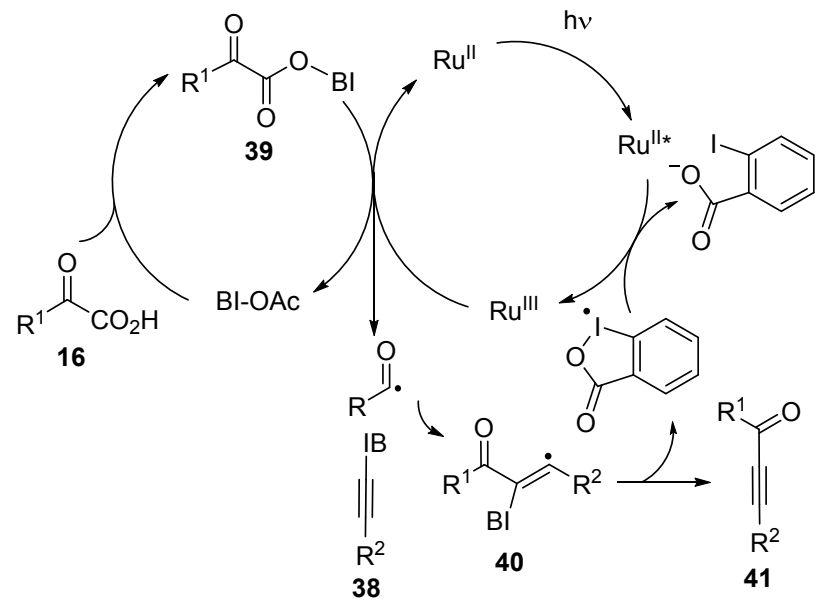

图式 4 炔基化反应机理

Scheme 4 Mechanism of the alkynylation

2015 年 Xiao 课题组 ${ }^{[62]}$ 报道了羧酸脱羧与炔烃的偶 联反应(Scheme 5).

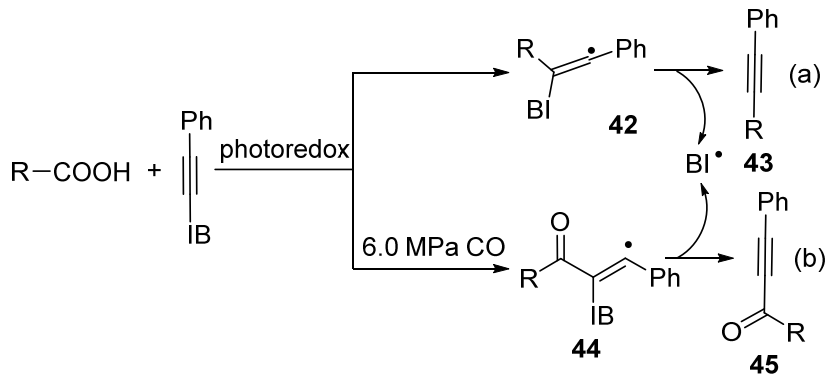

图式 5 羧酸与炔烃的偶联反应

Scheme 5 Coupling reaction of carboxylic acid and alkynes

与之前的报道工作不同的是该反应并不是过渡金 属参与炔基化的反应，而是在炔烃的端位引入高价碘基 才，这种官能化的炔烃在收到自由基进攻时能形成稳定 的烯基自由基 36, 再经 BI 自由基离去得到目标产物. 不仅如此，作者结合他们自己的前期研究工作 ${ }^{[63,64]}$ 对炔 烃的羰基化进行了研究，在 $60 \mathrm{MPa} \mathrm{CO}$ 氛围下同时实现 了脱羧和羰基化两种官能化的反应，验证了该方法广阔 的应用前景. Waser 等 $^{[65]}$ 几乎在同一时间也报道了该类 反应, 并提出了类似的反应机理, 也从另一方面证明了 该方法的可行性(Eq. 11).

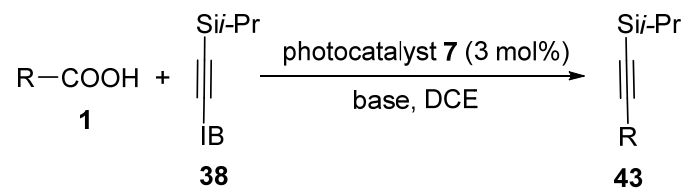


在类似领域, Wang 等 ${ }^{[66]}$ 随后报道了一种新型催化 体系催化酮酸脱羒合成羰基炔的反应, 该反应以高价碘 试剂 BI-OH 作为光催化剂, 以澳代的炔烃作为炔基化试 剂, 在太阳光下反应 $8 \mathrm{~h}$ 得到目标产物, 收率良好. 避免 了使用价格昂贵的金属催化剂, 反应的成本更低, 也是 今后光催化重要的发展方向.

\section{4 加成反应}

2013 年 Nishibayashi 等 ${ }^{[67]}$ 报道了对氨基苯乙酸与缺 电子烯烃的加成反应(Eq. 12).<smiles>[R]N([R])c1ccc(CC(=O)O)cc1</smiles><smiles>[R]CC(C[18O])Cc1ccc(N([R])[R])cc1</smiles>

该反应条件温和, 收率较高, 但是只有对位带有氨 基的苯乙酸才能在该体系中生成茮基自由基然后进行 加成反应, 因此该脱羧反应的底物类型有很大的局限 性.

2014 年 MacMillan 等 ${ }^{\left[{ }^{[8]}\right.}$ 报道了可见光催化羧酸类 化合物与缺电子烯烃的共轭加成反应应用在医药合成 中如普瑞巴林的合成等(Eq. 13).

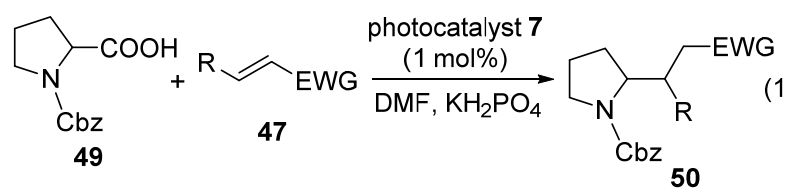

该反应是对经典迈克尔加成反应的拓展, 羧酸通过 氧化脱去 $\mathrm{CO}_{2}$ 生成自由基作为迈克尔供体与不同的烯 烃发生自由基加成反应, 反应条件简单, 底物范围广泛, 不仅 $\alpha$-氧, $\alpha$-氮类羧酸能参与反应, 而且烷基类的羧酸 也表现了较高的活性. 作为迈克尔受体的缺电子烯烃连 有酯基、醛基、砜基等官能团时都能得到相应的迈克尔 加成产物.

Overman 等 ${ }^{[69,70]}$ 则报道了在可见光催化体系, 叔碳 自由基与缺电烯烃加成反应生成新的季碳中心. 该反应 以邻苯二甲酰亚胺取代的叔醇作为叔碳自由基的前驱 体, 通过氧化作用脱去两分子 $\mathrm{CO}_{2}$ (Eq. 14).

后续该小组与 MacMillan 课题组合作, 对于这类型 反应又进一步做了拓展(Eq. 15) ${ }^{[71]}$. 用草酸盐取代的叔 醇代替了邻苯二甲酰亚胺取代的叔醇作为叔碳自由基

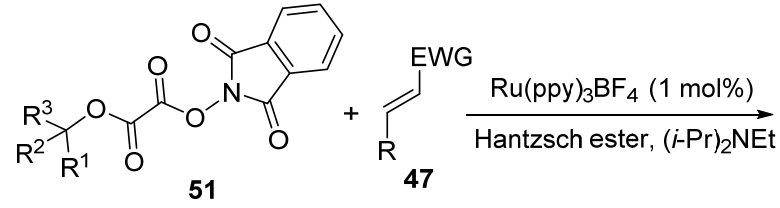<smiles>[R]C([R])C([R])([R])CC([R])([2H])C</smiles>

的前驱体, 取得了较好的效果. 草酸盐作为活化叔醇的 基团更为稳定，后处理更为简单.

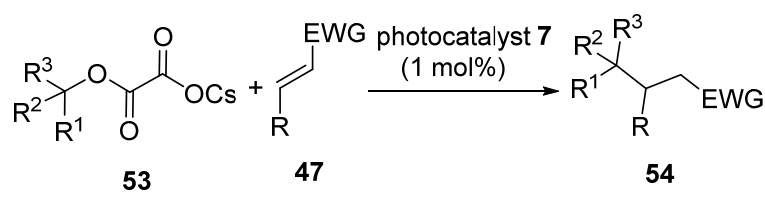

不仅如此, $\mathrm{Fu}$ 等 ${ }^{[72]}$ 将这类加成反应的底物进一步拓 展到酮酸, 在前面的几种反应类型中也提到酩酸参与脱 酸反应表现出了较高的活性, 也是一类新的酰基化反 应. 在该反应中, 酰基自由基对迈克尔受体的加成不仅 在毫克级反应中表现出了优良的反应性能, 而且在克级 反应中也得到了收率为 $85 \%$ 的加成产物(Eq. 16).
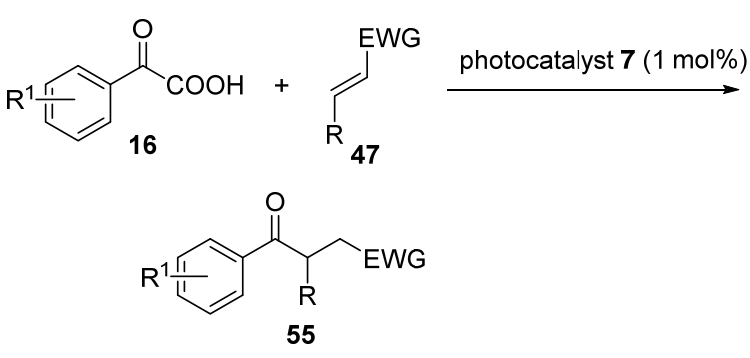

Wang 课题组 ${ }^{[73]}$ 结合他们在脱䊣炔基化反应方面的 工作, 将有光敏性的高价碘催化剂引入到加成反应体系 中合成了一系类吲哚衍生物. 发展了以高价碘试剂 BI$\mathrm{OAc}$ 光为光催化剂的催化体系. 该工作为合成该类吲哚 化合物提供了新的方法，进一步证明高价碘可以参与可 见光催化反应, 为后续该类试剂在光催化合成中应用奠 定了基础(Eq. 17).

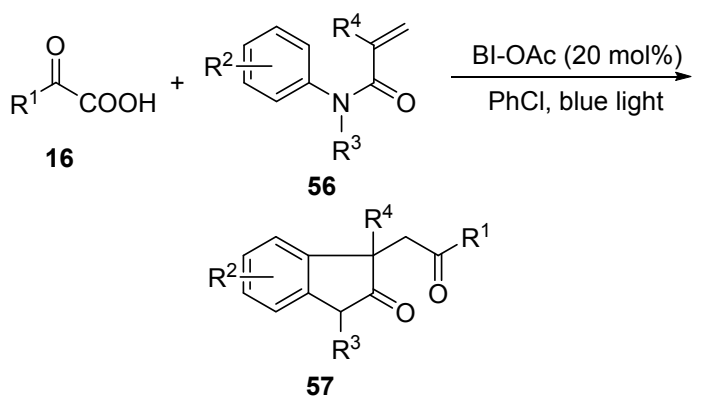




\section{5 烯丙基化反应}

2014 年 Tunge 等 ${ }^{[74]}$ 将过渡金属 Pd 引入光催化体系, 利用两种催化剂的协同作用实现了 $\alpha$-氨基羧酸烯丙基 酯分子内的脱羧烯丙基化反应(Scheme 6).

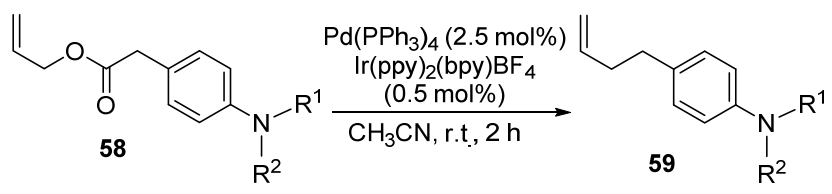<smiles>[R]C(CC=C)N([R])[R]</smiles>

60

61

图式 6 分子内的脱羧烯丙基化反应

Scheme 6 Intramolecular reaction of decarboxylative allylation

该反应克服了传统烯基化反应需要苛刻的反应条 件, 烯丙基化产物的形成是由脱羧后生成的茮基自由基 或 $\alpha$-氨基自由基与烯丙基自由基的偶联得到. 在后续的 研究工作中 ${ }^{[75]}$, 作者以带有缺电子保护基的 $\alpha$-氨基酸 烯丙基酯作为底物对这一类型的反应做了进一步的探 讨, 并深入讨论提出了两个相互竞争的反应机理 (Scheme 7). 在 Pd 催化剂和光催化剂的协同作用下, 如 果氧化脱羧生成的自由基足够稳定则以 $\mathrm{b}$ 路径为主生成 两个自由基然后偶联到目标产物, 如果生成的自由基不 够稳定则以 $\mathrm{a}$ 路径为主生成 Pd 中间体再经还原消除得 到目标产物. 作者分析认为, 当胺有缺电子保护基时生 成的自由基是不稳定的, 更倾向于生成金属中间体，这 也是当缺电子保护基的 $\alpha$-氨基酸烯丙基酯作为底物是 收率提高的原因所在.

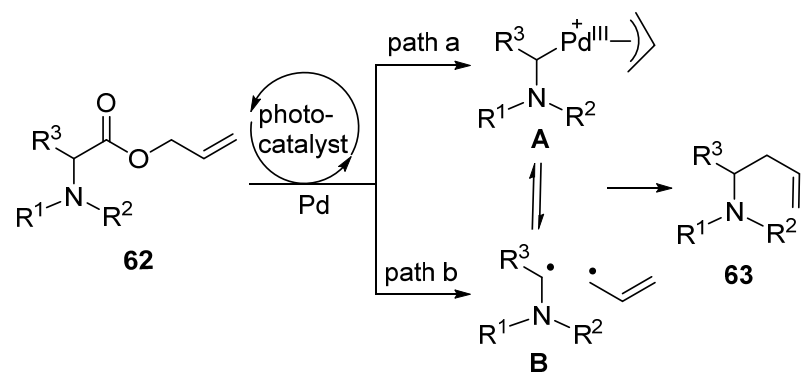

图式 7 脱羧烯丙基化的两种反应机理

Scheme 7 Two reaction mechanisms of decarboxylative allylation

\section{6 氟化反应}

氟化学是有机化学重要的一部分, 含氟有机分子被 广泛地应用在药物、功能材料方面 ${ }^{[76 ~ 78]}$. 其中, $\mathrm{C}_{\mathrm{sp}^{3}}-\mathrm{F}$
的构建较为困难, 近年来可见光催化的快速发展提供了 一种温和、高效地引入含氟官能团的方法 ${ }^{[79 ~ 83]} .2014$ 年 Sammis 等 ${ }^{[84]}$ 报道了首例光催化构建 $\mathrm{C}-\mathrm{F}$ 键的反应 (Eq. 18).

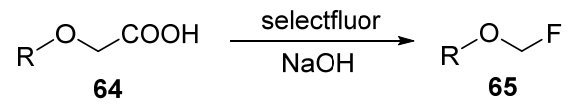

以选择性氟试剂(Selectfluor)作为氟源，同时其在反 应体系中又作为氧化剂参与到光氧化还原循环中. 芳氧 乙酸能被光催化剂氧化却不能被选择性氟氧化是该反 应成功的重要前提条件, 作者通过瞬态吸收光谱技术验 证了选择性氟首先氧化激发态 $\mathrm{Ru}$ 催化剂的氧化猝灭循 环. 该方法条件温和，收率良好，但是羧酸底物类型却 仅限于芳氧乙酸, 而随后 MacMillan 课题组 ${ }^{[85]}$ 报道的利 用选择性氟作为氟源进一步将底物范围扩大，不仅芳氧 乙酸能参与脱羧氟化反应，而且芐基羧酸、烷基羧酸等 均能成功实现氟化(Eq. 19).

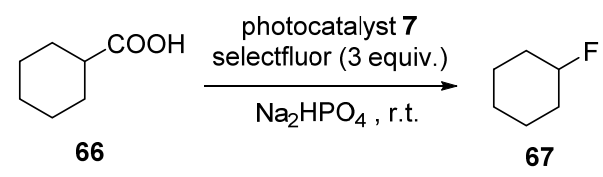

而 $\mathrm{Ye}$ 课题组 ${ }^{[86]}$ 则用了较为廉价的有机染料作为光 催化剂，同样能够完成脱羧氟化反应. 有机染料作为一 种绿色廉价光敏剂越来越受到关注, 而且相关报道中也 显示了其较高的活性, 也使可见光催化朝着绿色、低成 本的方向发展.

\section{7 脱羧还原反应}

2014 年, Wallentin 等 ${ }^{[87]}$ 报道了首例脱羧还原反应 (Eq. 20). 以有机鲇啶作为光敏剂, $\alpha$-氨基酸、 $\alpha$-差基氨基 酸等成功实现去羧基化合成了具有生物活性的不同仲 胺. 该反应不仅适用于带有保护基的 $\alpha$-氨基酸，脂肪 酸、苯乙酸等衍生物也同样能在该体系中脱去羧酸.

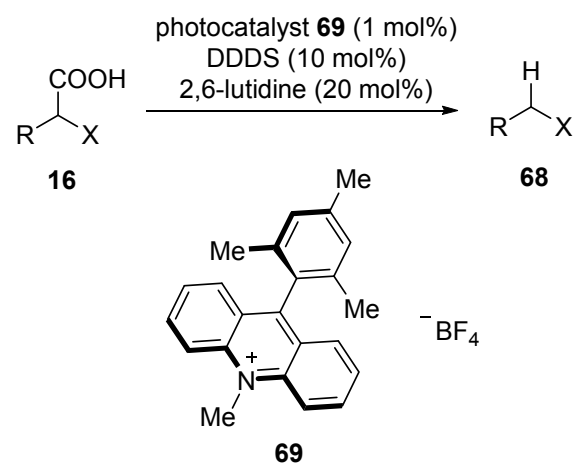

呋啶酯 69 在光作用下首先与 16 发生电子转移脱去 
$\mathrm{CO}_{2}$ 生成自由基中间体(Scheme 8), 同时催化剂被还原 成鲐啶自由基, 该自由基接着被硫自由基氧化, 完成催 化循环，同时生成的硫醇阴离子与 $\mathrm{H}^{+}$结合生成硫醇作 为氢供体与 70 作用生成目标产物. Nicewicz 等 ${ }^{[88 ~ 92]}$ 也 对这一类脱羧反应做了研究, 利用鲐啶与二苯二硫醚组 成的双催化体系, 以三氟乙醇作为溶剂, 增大了反应的 兼容性, 对参与反应的羧酸底物拓展到伯碳、叔碳、季 碳羧酸, 而且实现了丙二酸衍生物的双脱羧还原.

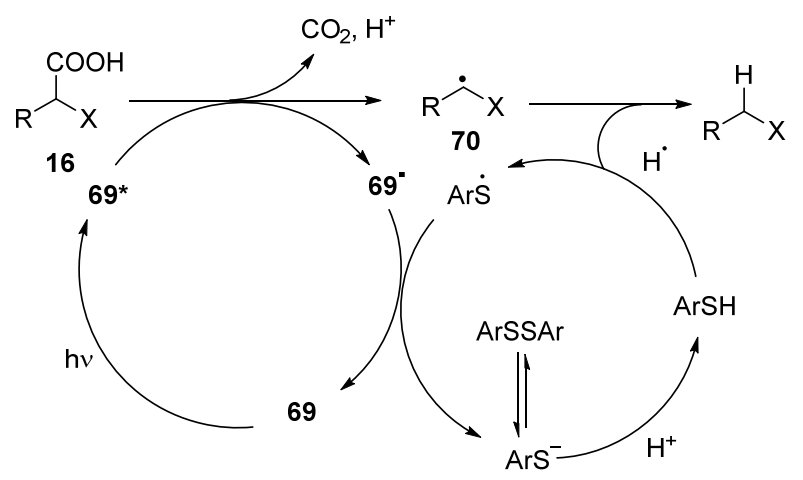

图式 8 羧酸还原反应

Scheme 8 Carboxylic acid reduction reaction

\section{8 酰胺化反应}

2014 年 Lei 等 ${ }^{[93]}$ 首次报道了酮酸脱羧与伯胺的偶联 反应(Eq. 21).

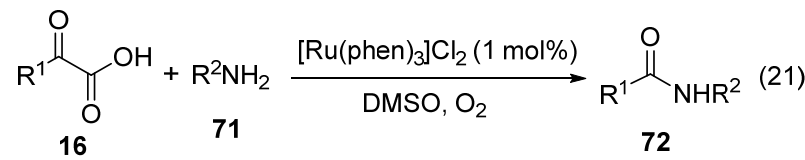

以廉价的 $\mathrm{O}_{2}$ 作为氧化剂, 而且发现 $\mathrm{O}_{2}$ 的活化作用 是该反应的决速步骤. 无论是酮酸还是伯胺, 底物范围 都十分广泛, 为合成一系列的酰胺提供了新的简单的方 法.

\section{9 总结与展望}

可见光介入的氧化脱羧与烯烃、炔烃等偶联反应实 现了不同的官能化反应, 为合成一些医药、功能材料等 提供了新的方法. 在上述的研究工作中, 不仅反应底物 被不断地拓展, 新的催化体系也不断被发现, 如将过渡 金属引入到光催化体系以及发展新的高效光催化剂如 高价碘试剂等. 随着研究的不断深入, 可见光催化会向 着更为简单、绿色的方向发展.

\section{References}

[1] Weaver, J. D; Recio, A; Grenning, A. J; Tunge, J. A. Chem. Rev. 2011, 111,1846
[2] Baudoin, O. Angew. Chem., Int. Ed. 2007, 46, 1373;

[3] Li, Z.; Jiang, Y. Y; Yeagley, A. A.; Bour, J. P.; Liu, L.; Chruma, J. J.; Fu, Y. Chem. Eur. J. 2012, 18, 14527.

[4] Burger, E. C.; Tunge, J. A. J. Am. Chem. Soc. 2006, 128, 10002.

[5] Yeagley, A. A.; Chruma, J. J. Org. Lett. 2007, 9, 2879.

[6] Xie, J.; Jin, H.; Xu, P.; Zhu, C. Tetrahedron Lett. 2014, 55, 36.

[7] Chen, J.; Cen, J.; Xu, X. L.; Li, X. N. Catal. Sci. Technol. 2016, 6, 349.

[8] Nicewicz, D. A.; MacMillan, D. W. C. Science 2008, 322, 77.

[9] Nagib, D. A.; Scott, M. E.; MacMillan, D. W. C. J. Am. Chem. Soc. 2009, 131, 10875.

[10] Pirnot, M. T.; Rankic, D. A.; Martin, D. B. C.; MacMillan, D. W. C. Science 2013, 339, 1593.

[11] McNally, A.; Prier, C. K.; MacMillan, D. W. C. Science 2011, 334, 1114.

[12] Shih, H. W.; Wal, M. N. V.; Grange, R. L.; MacMillan, D. W. C. J. Am. Chem. Soc. 2010, 132, 13600.

[13] Jeffrey, J.; Petronijevic, F. R.; MacMillan, D. W. C. J. Am. Chem. Soc. 2015, 137, 8404.

[14] Ischay, M. A.; Anzovino, M. E.; Du, J.; Yoon, T. P. J. Am. Chem. Soc. 2008, 130, 12886.

[15] Du, J.; Yoon, T. P. J. Am. Chem. Soc. 2009, 131, 14604.

[16] Lin, S.; Ischay, M. A.; Fry, C. G.; Yoon, T. P. J. Am. Chem. Soc. 2011, 133, 19350.

[17] Lu, Z.; Yoon, T. P. Angew. Chem., Int. Ed. 2012, 51, 10329.

[18] Blum, T. R.; Zhu, Y.; Nordeen, S. A.; Yoon, T. P. Angew. Chem., Int. Ed. 2014, 53, 11056.

[19] Narayanam, J. M. R.; Tucker, J. W.; Stephenson, C. R. J. J. Am Chem. Soc. 2009, 131, 8756.

[20] Narayanam, J. M. R.; Stephenson, C. R. J. Chem. Soc. Rev. 2011, $40,102$.

[21] Condie, A. G.; Gonzalez-Gomez, J. C.; Stephenson, C. R. J. J. Am. Chem. Soc. 2010, 132, 1464.

[22] Freeman, D. B.; Furst, L.; Condie, A. G.; Stephenson, C. R. J. Org Lett. 2012, 14, 94

[23] Xuan, J.; Cheng, Y.; An, J.; Lu, L. Q.; Zhang, X. X.; Xiao, W. J. Chem. Commun. 2011, 47, 8337.

[24] Zou, Y. Q.; Guo, W.; Liu, F. L.; Lu, L. Q.; Chen, J. R.; Xiao, W. J. Green Chem. 2014, 16, 3787.

[25] Xuan, J.; Xia, X. D.; Zeng, T. T.; Feng, Z. J.; Chen, J. R.; Lu, L. Q.; Xiao, W. J. Angew. Chem., Int. Ed. 2014, 53, 5633.

[26] Xie J.; Xu P.; Li, H.; Xue, Q.; Jin, H.; Cheng, Y.; Zhu, C. Chem. Commun. 2013, 49, 5672.

[27] Xuan, J.; Zhang, Z. G.; Xiao, W. J. Angew. Chem., Int. Ed. 2015, $54,15632$.

[28] Zuo, Z.-W.; MacMillan, D. W. C. J. Am. Chem. Soc. 2014, 136, 5257

[29] Lloyd-Jones, G. C.; Ball, L. T. Science 2014, 345, 381.

[30] Terrett, J. A.; Cuthbertson, J. D.; Shurtleff1, V. W.; MacMillan, D. W. C. Nature 2015, 524, 330 .

[31] Hopkinson, M. N.; Sahoo, B.; Glorius, F. Adv. Synth. Catal. 2014, 356,2794

[32] Tellis, J. C. Primer, D. N.; Molander, G. A. Science 2014, 345, 433.

[33] Cai, S.Y.; Yang, K.; Wang, D. Z. Org. Lett. 2014, 16, 2606.

[34] Choi, S.; Chatterjee, T.; Choi, W. J.; You, Y.; Cho, D. E. J. ACS Catal. 2015, 5, 4796.

[35] Shu, X. Z.; Zhang, M.; He, Y.; Frei, H.; Toste, F. D. J. Am. Chem. Soc. 2014, 136, 5844.

[36] Anderson, A. J. M.; Koehi, J. K. J. Am. Chem. Soc. 1970, 92, 1651.

[37] Miller, J. A.; Nelson, J. A.; Byrne, M. P. J. Org. Chem. 1993, 58, 18.

[38] Samuel, C.; Simone, T.; Mueller, J.; Jerome, L. Angew. Chem., Int. 
Ed. 2007, 46, 2082.

[39] Burger, E. C.; Tunge. J. A. Chem. Commun. 2005, 2835.

[40] Zuo, Z.; Ahneman, D. T.; Chu, L.; Terrett, J. A.; Doyle, A. G.; MacMillan, D. W. C. Science 2014, 345, 437.

[41] Oderinde, M. S.; Varela-Alvarez, A.; Aquila, B.; Robbins, D. W.; Johannes, J. W. J. Org. Chem. 2015, 80, 7642.

[42] Chu, L.; Lipshultz, J. M.; MacMillan, D. W. C. Angew. Chem., Int. Ed. 2015, 54, 7929.

[43] Cheng, W. M.; Shang, R.; Yu, H. Z.; Fu, Y. Chem. Eur. J. 2015, 21, 13191.

[44] Noble, A.; McCarver, S. J.; MacMillan, D. W. C. J. Am. Chem. Soc. 2015, 137, 624.

[45] Zou, Y. Q.; Duan, S. W.; Meng, X. G.; Hu, X. Q.; Gao, S.; Chen, J. R. Xiao, W. J. Tetrahedron 2012, 68, 6914.

[46] Tucker, J. W.; Narayanam, J. M. R.; Krabbe, S. W.; Stephenson, C. R. J. Org. Lett. 2010, 12, 368.

[47] Furst, L.; Matsuura, B. S.; Narayanam, J. M. R.; Tucker, J. W.; Stephenson, C. R. J. Org. Lett. 2010, 12, 3104.

[48] Furst, L.; Narayanam, J. M. R.; Stephenson, C. R. J. Angew. Chem., Int. Ed. 2011, 50, 9655.

[49] Ju, X.; Liang, Y.; Jia, P.; Li, W.; Yu, W. Org. Biomol. Chem. 2012, $10,498$.

[50] Maity, S.; Zheng, N. Angew. Chem., Int. Ed. 2012, 51, 9562.

[51] Chen, M.; Huang, Z. T.; Zheng, Q. Y. Chem. Commun. 2012, 48, 11686.

[52] Fu, W.; Xu, F.; Fu, Y.; Zhu, M.; Yu, J.; Xu, C.; Zou, D. J. Org. Chem. 2013, 78, 12202.

[53] Zhang, P.; Xiao, T.; Xiong, S.; Dong, X.; Zhou, L. Org. Lett. 2014, $16,3294$.

[54] Gu, L.; Jin, C.; Liu, J.; Zhang, H.; Yuan, M.; Li, G. Green Chem. 2016, 18, 1201.

[55] Noble, A.; MacMillan, D. W. C. J. Am. Chem. Soc. 2014, 136, 11602

[56] Huang, H.; Jia, K.; Chen, Y. Angew. Chem., Int. Ed. 2015, 54, 1881.

[57] Rueping, M.; Koenigs, R. M.; Poscharny, K.; Fabry, D. C. Leonori, D.; Vila, C. Chem. Eur. J. 2012, 18, 5170.

[58] Adrian, T. A.; Matthew, N. H.; Basudev, S.; Frank, G. Chem. Sci. 2016, 7, 89

[59] Kim, S.; Martin, J. R.; Toste, F. D. Chem. Sci. 2016, 7, 85.

[60] Yang, J.; Zhang, J.; Qi, L.; Hu, C.; Chen, Y. Chem. Commun. 2015, $51,5275$.

[61] Huang, H.; Zhang, G.; Chen, Y. Angew. Chem., Int. Ed. 2015, 54, 7872 .

[62] Zhou, Q. Q.; Guo, W.; Ding, W.; Wu, X.; Chen, X.; Lu, L. Q.; Xiao, W. J. Angew. Chem., Int. Ed. 2015, 54, 11196.

[63] Guo, W.; Lu, L. Q.; Wang, Y.; Wang, Y. N.; Chen, J. R.; Xiao, W. J. Angew. Chem., Int. Ed. 2015, 54, 2265.

[64] Majek, M.; Wangelin, A. J. Angew. Chem., Int. Ed. 2015, 54, 2270.

[65] Vaillant, F. L.; Courant, T.; Waser, J. Angew. Chem., Int. Ed. 2015, 54,11200 .

[66] Tan, H.; Li, H.; Ji, W.; Wang, L. Angew. Chem., Int. Ed. 2015, 54,
8374.

[67] Miyake, Y.; Nakajima, K.; Nishibayashi, Y. Chem. Commun. 2013, 49, 7854 .

[68] Chu, L.; Ohta, C.; Zuo, Z.; MacMillan, D. W. C. J. Am. Chem. Soc. 2014, 136, 10886.

[69] Lackner, G. L.; Quasdorf, K. W.; Overman, L. E. J. Am. Chem. Soc. 2013, 135,15342 .

[70] Lackner, G. L.; Quasdorf, K. W.; Pratsch, G.; Overman, L. E. J. Org. Chem. 2015, 80, 6012.

[71] Nawrat, C. C.; Jamison, C. R.; Slutskyy, Y.; MacMillan, D. W. C. J. Am. Chem. Soc. 2015, 137, 11270.

[72] Wang, G. Z.; Shang, R.; Cheng, W. M.; Fu, Y. Org. Lett. 2015, 17, 4830.

[73] Ji, W.; Tan, H.; Wang, M.; Li, P.; Wang, L. Chem. Commun. 2016, $52,1201$.

[74] Lang, S. B.; O'Nele, K. M.; Tunge, J. A. J. Am. Chem. Soc. 2014, $136,13606$.

[75] Lang, S. B.; ONele, K. M.; Douglas, J. T.; Tunge, J. A. Chem. Eur J. 2015, 21,18589

[76] Müller, K.; Faeh, C.; Diederich, F. Science 2007, 317, 1881.

[77] Neumann, C. N.; Ritter, T. Angew. Chem., Int. Ed. 2015, 54, 3216.

[78] Purser, S.; Moore, P. R.; Swallow, S.; Gouverneur, V. Chem. Soc. Rev. 2008, 37, 320.

[79] Nagib1, D. A.; MacMillan, D. W. C. Nature 2011, 480, 224.

[80] Ye, Y.; Sanford, M. S. J. Am. Chem. Soc. 2012, 134, 9034.

[81] Sahoo, B.; Li, J. L.; Glorius, F. Angew. Chem., Int. Ed. 2015, 54, 11577.

[82] Su, Y. M.; Hou, Y.; Yin, F.; Xu, Y. M.; Li, Y.; Zheng, X.; Wang, X. S. Org. Lett. 2014, 16, 2958

[83] Tomita, R.; Yasu, Y.; Koike, T.; Akita, M. Angew. Chem., Int. Ed. 2014, $53,7144$.

[84] Wolf, M. O.; Sammis, G. M.; Paquin, J. F. J. Am. Chem. Soc. 2014, 136, 2637.

[85] Ventre, S.; Petronijevic, F. R.; MacMillan, D. W. C. J. Am. Chem. Soc. 2015, 137, 5654.

[86] Wu, X.; Meng, C.; Yuan, X.; Jia, X.; Qian, X.; Ye. J. Chem. Commun. 2015, 51, 11864.

[87] Cassani, C.; Bergonzini, G.; Wallentin, C. J. Org. Lett. 2014, 16, 4228.

[88] Nguyen, T. M.; Nicewicz, D. A. J. Am. Chem. Soc. 2013, 135, 9588.

[89] Nguyen, T. M.; Manohar, N.; Nicewicz, D. A. Angew. Chem., Int. Ed. 2014, 53, 6198.

[90] Romero, N. A.; Nicewicz, D. A. J. Am. Chem. Soc. 2014, 136, 17024.

[91] Morse, P. D.; Nicewicz, D. A. Chem. Sci. 2015, 6, 270.

[92] Griffin, J. D.; Zeller, M. A.; Nicewicz, D. A. J. Am. Chem. Soc. 2015, 137, 11340.

[93] Liu, J.; Liu, Q.; Yi, H.; Qin, C.; Bai, R.; Qi, X.; Lan, Y. Lei, A. Angew. Chem., Int. Ed. 2014, 53, 502. 